\title{
COVID-19 AND THYROID DISEASE: CLINICAL COURSE AND PROGNOSIS
}

\author{
Daria Korchagina, Iegor Korchagin
}

The aim is to analyze current scientific data on the prevalence of thyroid dysfunction in patients with COVID-19 and to evaluate the relationship between possible complications of COVID-19 and vaccination.

Materials and methods. Open digital archive of journal articles on biomedical and biological sciences of the National Institutes of Health (USA), developed by the National Center for Biotechnological Information of the National Medical Library (USA) - PubMed, Google Academy and Academic Journals.

Results. The development of thyroid diseases in SARS-CoV-2 may be associated with various mechanisms of its damage, including an excessive immune response, infection-induced immunodeficiency, or direct cell damage due to significant tissue tropism and high affinity of SARS-CoV-2 to thyroid tissue. Possible mechanisms of formation of postvaccination dysfunction of the thyroid gland are proposed.

Conclusions. Thyroid hormone deficiency is associated with an increased risk of adverse events and in-hospital mortality of COVID-19 and depended on the age of the patient. The severe course of Covid-19 is characterized by an increase in the prevalence of TT secondary to destructive or inflammatory thyroiditis. Thyroid-related thyrotoxicosis exacerbates the clinical course of comorbidities and long-term consequences, such as autoimmune hypothyroidism, which occurs in patients of all ages and with any severity of COVID-19.

The prevalence of TD in patients with COVID-19 varies from 13 to $64 \%$. The presence of thyroid dysfunction is positively correlated with the clinical severity of COVID-19. Patients with confirmed thyroid disease should receive COVID-19 vaccine to reduce the risk of morbidity and mortality from COVID-19 infection

Keywords: thyroid pathology, hypothyroidism, thyrotoxicosis, thyroiditis, COVID-19, vaccination, consequences

How to cite:

Korchagina, D., Korchagin, Ie. (2022). COVID-19 and thyroid disease: clinical course and prognosis. ScienceRise: Medical Science, 1 (46), 4-11. doi: http://doi.org/10.15587/2519-4798.2022.252809

(C) The Author(s) 2022

This is an open access article under the Creative Commons CC BY license hydrate

\section{Introduction}

From the end of 2019, the world is fighting the SARS-CoV-2 pandemic, which manifests itself as a mild flu-like or asymptomatic form in most patients, but may also be accompanied by multiple organ dysfunction (acute respiratory syndrome, septic shock, renal failure) [1-3] due to cardiovascular, respiratory, nervous and gastrointestinal systems, the dysfunction of which significantly impairs recovery after COVID-19. Patients with COVID-19 suffer from high mortality not only due to respiratory failure, but also due to cardiovascular collapse and disseminated intravascular coagulation [4-6]. Reports of extrapulmonary and atypical manifestations of COVID-19 indicate hemoptysis, cardiac, ocular and cutaneous manifestations, as well as venous and arterial thrombosis [7]. Specialists who studied the clinical course and risk factors for mortality in adult inpatients with COVID-19 found concomitant pathology in $48 \%$ of patients in the form of hypertension (30\% of patients), diabetes (19\% of patients) and coronary heart disease. About $90 \%$ of patients with COVID-19 pneumonia had increased coagulation activity, as determined by an increase in the concentration of D-dimer, the value of which when entering more than $1 \mu \mathrm{g} / \mathrm{ml}$ led to a higher chance of death in hospital. The authors consider the occurrence of sepsis in more than half of the subjects in whom no bacterial pathogens were detected on admission, due directly to SARS-CoV-2 infection [8].

Key factors in SARS-CoV-2 infection are angiotensin-converting enzyme 2 (ACE2), which serves as a "receptor" that allows the virus to enter thyroid cells, and the transmembrane protease that SARS-CoV-2 virus uses to penetrate cells $[9,10]$. In addition, ACE2, the SARS-CoV-2 receptor, is expressed on myocytes and vascular endothelial cells, so there is at least a theoretical potential for direct virus damage to the heart [8].Elderly age, male gender and comorbidities are the main risk factors for more severe COVID-19 and worse treatment outcomes [8, 11, 12].

Concomitant pathology increases the frequency of hospitalization in the intensive care unit (ICU) and mortality from COVID-19 in elderly patients $[4,13]$, and is considered a risk factor for increased oxygen demand, artificial lung ventilation [ALV] [14].

But among the most common diseases associated with COVID-19 (hypertension, diabetes, coronary heart disease, cerebrovascular disease), which complicate the course and prognosis of the disease, thyroid pathology in patients with pre-existing thyroid problems [15], or in which thyroid problems occurred after infection, not mentioned $[6,8,10,16]$. 
The aim of the research was to analyze scientific data on the prevalence of thyroid dysfunction in patients with COVID-19 and evaluate the relationship between possible complications of COVID-19 and vaccination.

\section{Materials and methods}

Open digital archive of journal articles on biomedical and biological sciences of the National Institutes of Health (USA), developed by the National Center for Biotechnological Information of the National Medical Library (USA) - PubMed, Google Academy and Academic Journals.

\section{Research results}

With sufficient study of the clinical course, prognostic markers of inflammation and complications of COVID-19, there are conflicting assumptions that thyroid disease may increase the risk of severe COVID-19 [17-19]. These assumptions are refuted by claims about SARS-CoV-2 as the cause of only short-term and reversible thyroid dysfunction (TD), which does not affect the progression of COVID-19 [20].

A systematic review with significant heterogeneity of data, involving 1,237 patients with COVID-19, found that most patients were euthyroid with normal TSH levels (44 to $94 \%$ of patients). A study of the acute effects of COVID-19 on thyroid function in a cohort of 334 patients also found that $86.6 \%$ of patients with COVID-19 had euthyroidism [21, 22].

Routine assessment of thyroid function during hospitalization for COVID-19 is not recommended by the World Health Organization (2020) Clinical Management of Severe Acute Respiratory Infection (SARI) guidelines when COVID-19 disease is suspected: interim guidance. At the same time, they draw attention to the possibility of worsening the course of pre-existing TD or de novo thyroid pathology caused by the infection itself [23].

The prevalence of TD in patients with COVID19 , defined as abnormal thyroid function tests, ranges from 13 to $64 \%$. A positive correlation was found between TD and the clinical severity of COVID-19 $[21,24]$. At the same time, fluctuations in TD in COVID-19 from thyrotoxicosis (TT) to complete suppression of thyroid function have been reported [14, $20,24]$. Determination of thyroid function in 60 hospitalized patients with COVI D-19 without a history of thyroid disease, of which $43.3 \%$ had a mild, $26.7 \%-$ moderate and $30 \%$ - severe COVID-19, showed the presence of one or multiple thyroid dysfunction in $35 \%$ of patients. The most common of these were low TSH levels $(18.33 \%)$ and signs of thyroiditis $(9.1 \%)$ [25].

According to studies, hypothyroidism (HT) was the third most common disease among patients with COVID 19, found the dependence of the prevalence of HT on the severity of COVID-19, finding HT in $2.7 \%$ of patients in a small sample of 365 surveyed, and following an increase to $3.6 \%$ in severe patients and up to $4.85 \%$ in patients on mechanical ventilation [14]. In the list of comorbidities in [17], GT also ranks third in prevalence $(18.2 \%)$ after diabetes $(50.4 \%)$ and hyper- tension [21, 28]. Bakshi, S.S., \& Kalidoss, V.K. (2021) in a search for a link between HT and COVID 19 in 456 patients with positive RT-PCR (polymerase chain reaction reverse transcription) found a lower proportion of HT $(25.3 \%)$, but in $7(1.5 \%)$ of them was the only comorbid disease [11].

It has been suggested that changes in COVID-19 in patients without a history of TD may include: impaired TSH synthesis due to virus-related pituitary gland [26]; atypical subacute thyroiditis (SAT), which depends on the spread of the virus or excessive production of cytokines, including a destructive process with irreversible damage to the gland; non-thyroid disease syndrome (NTDS) is not caused by COVID-19 infection, but by a very severe course of the disease [27].

It is believed that COVID-19 and TD may mutually exacerbate the burden of the disease [28], separately pointing to the dangerous comorbidity of HT and TT as a threat of severe cardiovascular complications. Other experts [27] have found that most patients with previously known thyroid disease are not at higher risk of SARSCoV-2 infection or risk of hospitalization for more severe infections. Thus, in a study aimed at studying the relationship between susceptibility, morbidity and mortality from COVID-19 in patients with HT, TT, Graves' disease (GD) and a history of thyroiditis in 8070 patients with COVID-19 and 32,280 members of the control group, found no such connection with the studied thyroid pathology [10].

A systematic review of the potential effects of COVID-19 on the thyroid gland includes TT, low T3 syndrome, SAT, and NTDS on ICU hospitalization. The presence of TD has been shown not to increase the risk of SARS-CoV-2 infection, and patients with TD do not require further follow-up adapted to COVID-19 [29]. Based on the results of a survey of 3,703 patients with laboratory-confirmed COVID-19, of whom about $7 \%$ had HT and received hormone replacement therapy, van Gerwen, M. et al. (2020) argue that HT is not associated with an increased risk of hospitalization, mechanical ventilation or death, and patients with HT do not require additional precautions or counseling [15].

21 cases $(5.4 \%)$ of HT were identified among 390 patients with COVID-19, $90 \%$ of whom were aged 50 years and older. Among the deceased 60 (15.3\%) of the total number of patients and $4(19 \%)$ patients with HT, no significant differences were found [30].

It is believed that TD affects the outcome of the disease by increasing mortality in critical diseases such as acute respiratory syndrome, which is a leading complication of COVID-19 [31]. Patients with COVID-19 and uncompensated TD (i.e., biochemically confirmed hypo- and hyperthyroidism) were found to have a higher risk of complications and death compared to patients without TD and a positive correlation between TD and disease severity [4, 19], which contradicts in another study [25] concluded that there was no significant difference in the parameters of TD depending on the severity of COVID-19. There are no differences between patients with hypo- and hyperthyroidism and euthyroid patients in the clinical outcome of COVID-19 (mortality, hospitalization for ICU and use of mechanical ventilation) [14]. To a greater extent, the course of the disease is deter- 
mined by whether patients received hormone replacement therapy and whether they have achieved euthyroidism [10]. Well-controlled hypo - and hyperthyroidism are not associated with an increased risk of COVID - 19 infection or its severity [32]. Unexpected results obtained from the analysis of the Brazilian COVID-19 registry Hypothyroidism does not lead to a worse prognosis in the COVID-19 registry: findings from the Brazilian COVID19 registry. To determine whether previous thyroid disease affected COVID-19, the clinical characteristics and treatment outcomes of 7,762 patients aged about 70 years, with and without COVID-19, were compared. It was found that the length of hospital stay ( 8 vs. 9 days; $\mathrm{p}=0.029)$, the need for mechanical ventilation $(25.4 \% \mathrm{vs}$. $33.1 \%$; $=0.006$ ) were lower in patients with HT. They also showed a tendency to reduce in-hospital mortality (22.1\% vs. $27.0 \% ; \mathrm{p}=0.062)$. The conclusion seems somewhat unexpected given that with the same treatment and comparable comorbidity in the groups, coronary heart disease and chronic kidney disease were more common in the HT group [33].

Currently, the American Thyroid Association (ATA) has no specific guidelines for patients with previously diagnosed HT and advises them to continue taking COVID-19 as prescribed [34].

The development of thyroid disease in severe SARS-CoV-2 may be associated with various mechanisms of its damage, including excessive immune response, immunodeficiency due to infection, or direct cell damage due to significant tissue tropism and high affinity of SARS-CoV-2 [26]. In this process, the role of thyroid hormones in the regulation of the innate immune response is crucial [35]. Excess or deficiency of thyroid hormones, observed in thyroid disease, leads to a violation of this function, which makes the greatest contribution to the pathogenesis of COVID-19. This is evidenced by an increase in severe infection (neutrophils, monocytes, macrophages, etc.] [36] and the level of proinflammatory cytokines TNF- $\alpha$ and IL-6, which are characteristic of thyroid disease, and in patients with severe COVID-19 correlate with the development of severe consequences [24, 37].

The mechanisms of potential association between SARS-CoV-2 and TD have not been definitively elucidated [8, 19, 38-41]. Literary research provides a lot of evidence that the hypothalamus-pituitary-thyroid gland may be relevant targets for SARS-CoV-2 [2]. In turn, referring to the presence of TD in patients with COVID19 , in addition to the possibility of dysfunction is likely dysfunction of the hypothalamic-pituitary-adrenal axis [31]. It has also been shown that SARS-CoV-2 provokes autoimmune diseases [9, 42].

To date, TT, GT, NTDS, as well as subacute, painless, postpartum and autoimmune thyroiditis and atypical forms of thyroiditis have been registered as complications of COVID-19 [2, 5, 31, 43, 44].

In two cases of TT, which were diagnosed 1 and 2 months after the clinical onset of COVID-19. Both patients were initiated on thiamazole and propranolol therapy to improve thyroid symptoms and function. The clinical picture, increased thyrotropin uptake, and positive antibodies to TSH receptors have shown compatibil- ity with a diagnosis of Graves' disease (autoimmune hyperthyroidism) [45].

It is known that in addition to SARS-CoV-2, several other respiratory viruses, including Coxsackie virus, mumps, Epstein-Barr virus, cytomegalovirus, and influenza virus, could be potent triggers for SAT, which differs from the typical clinical course. These patients were called "atypical" because thyroid pain and edema could be absent, and focal hypoechoic areas, decreased $99 \mathrm{mTc}$ uptake, and autologous autoantibody negatives were identified that distinguished them from typical SAT and led to a new concept of "atypical SAT" [42, 44].

The identified affinity of SARS-CoV-2 for the thyroid gland draws attention to the potential risk of developing GD [9, 45] and Hashimoto's thyroiditis [26], but SAT -inflammatory thyroid disease of viral or postviral origin [24, 29, 45-47] was the most common thyroiditis associated with COVID-19 [2, 9, 48]. Previous viral infection, which occurred approximately 2-6 weeks earlier, is considered a factor that provokes SAT [1, 44, 46]. The natural course of SAT usually includes the initial thyrotoxic phase [3], followed by the GT phase followed by recovery to the euthyroid state, i.e., SARSCoV-2 could cause reversible TD, including subclinical and atypical thyroiditis [1,20]. Patients usually have an increase in serum T3 and T4 levels along with a decrease in TSH levels [31, 49]. TT in SAT usually lasts for 3 months [49].

A clinical case of atypical PR is a case of TT in a 69-year-old woman with a history of euthyroidism during hospitalization for COVID-19. Nasopharyngeal smear for SARS-CoV-2 was positive, chest CT showed bilateral areas of frosted glass, typical of interstitial SARSCoV-2 pneumonia. The clinical picture, ultrasound characteristics, high levels of thyroglobulin in the serum and the absence of autoantibodies to the thyroid gland indicated a destructive process in the thyroid gland, compatible with the diagnosis of SAT, against which naturally developed TT [23].

Laboratory diagnosis, thyroid scintigraphy, and SAT with TT six weeks after SARS-COV-2 infection, whose symptoms were mild and did not require treatment, have been described in detail [47].

Studies have brought a positive approach to whether SAT is an underestimated manifestation of SARS-CoV-2 infection. The first shows the manifestations of SAT in 3 patients with COVID-19 16-36 days after the end of the disease: increased levels and markers of inflammation and signs of destructive TT, significantly reduced in a few days after treatment (prednisolone), and 6 weeks after the onset of SAT disappeared simultaneously with the normalization of levels of inflammatory markers. In the recovery period, 2 patients had euthyroidism, and 1 had subclinical HT [50]. Another clinical case reported a mild case of SAT that developed 6 weeks after the onset of SARS-COV-2 infection and ended in euthyroidism and normalization of inflammation with corticosteroid therapy for 4 weeks [51].

A review of the literature has shown that the most common thyroid hormone levels in patients with severe COVID-19 are similar to those present in NTDS [45]. The pathogenesis of NTDS, characterized by decreased 
serum T3 levels, a persistent critical disease, is complex and involves inhibition of hypothalamic thyrotropinreleasing hormone, which causes a steady decrease in TSH secretion despite its low plasma levels. There is a positive correlation between low mean T3 and clinical severity of COVID-19 [43, 52]. Low levels of T3 on admission to the hospital independently indicate the severity of COVID-19 [53], as well as have a negative correlation with the length of hospital stay and the level of CRP [54]. It has been shown that SARS-CoV-2 can directly affect thyroid function, which may exacerbate pre-existing autoimmune thyroid disease. Low levels of T3 are associated with systemic inflammation and may be prognostic [24], with decreased $\mathrm{SpO}_{2}$ and lymphocyte counts may be potential indicators of severe COVID-19, regardless of comorbidities, the elderly, and sex [55].

The degree of NTDS is related to the prognosis of the disease [56], with an increased risk of 30-day mortality and hospitalization in the ICU in patients with severe disease and differs significantly between patients who "survived or did not survive": $29.1 \%$ vs. $71,9 \%$, $\mathrm{P}<0.001$ [53] The moderate decrease in TSH levels found in the cohort of 334 patients with COVID-19 was assessed by the authors as NTDS-compliant. baseline [22].

In 191 patients hospitalized with COVID-19 with mild $(84.3 \%)$, moderate $(12.6 \%)$, and severe $(3.1 \%)$ course of the disease, isolated low T3 was considered as NTDS, there was a tendency to decrease T3 with increasing severity of COVID-19 $(\mathrm{P}=0.032)$ [24].

Comparison of thyroid function in patients with pneumonia COVID-19 (1 g) with patients treated in the ICU for acute respiratory syndrome ( $2 \mathrm{~g})$, showed a statistically significant decrease in T3 and TSH in them compared with the control "euthyroid" group. In group 2, a further statistically significant decrease in the level of T3 and TSH was found [43].

The significant decrease in TSH levels in almost half of the 50 patients with COVID-19 found, together with the decrease in serum T3, was rightly considered by them as an indicator of the severity of COVID-19 due to the release of systemic anti-inflammatory cytokines or direct viral effects on the pituitary or thyroid gland [57]. Baldelli R. et al. (2021) have shown that serum T3 and TSH levels are lower in patients with more severe symptoms [40]. In a study of TD conducted by Dabas, A. et al. (2021) in 84 patients out of 164 with COVID-19, found only a decrease in serum T3 mild, moderate and severe, which to varying degrees determined the increased inflammation and risk of death [58].

The study, which included a total of 127 patients, of whom 116 survived and 11 did not survive, was that serum T3 levels were lower in non-surviving COVID-19 patients with moderate to critical severity, and low T3 is associated with an increased risk of nosocomial mortality from COVID-19 [41].

The recognition that the presence of low T3 syndrome is associated with poor clinical outcomes in critically ill patients has not yet led experts to conclude whether triiodothyronine supplements may be a tool to reduce this burden [20].

Severe Covid-19 is characterized by an increase in the prevalence of secondary TT in relation to destructive or inflammatory thyroiditis $[3,26] .20 .2 \%$ of patients hospital- ized for severe Covid-19 have clinical $(10.8 \%)$ or subclinical $(9.4 \%)$ TT [3]. It has been shown that 58 of $287(20 \%)$ patients with COVID-19 hospitalized in the ICU show TT caused by systemic inflammation or immune activation caused by COVID-19, and indicate a strong inverse correlation between serum IL-6 levels and TSH, which partly contributes to the contribution of inflammatory destruction of the thyroid gland in increasing the level of its hormones [59]. The increase in interleukin-6 concentration is secondary to the "cytokine storm" observed in severe SARS-CoV2 infections [56]. In a study by Lania, A. (2020) to examine possible changes in thyroid function during a cytokine storm, thyroid function was assessed in 193 patients with COVID-19 hospitalized in the ICU and found that COVID19 may be associated with high-risk TT due to systemic immune activation caused by SARS-CoV-2 infection. This is in line with CaronP's view (2020) that during severe forms of SARS-CoV-2 infection, a "cytokine storm" may be associated with inflammatory or destructive thyroiditis with TT [26].

Published clinical cases of 2 patients without a history of TD who developed severe TT of varying severity ("cytokine storm" and "impending cytokine storm"), possibly caused by SARS-CoV-2 infection and suggested SARS-CoV infection- 2 as a causative agent of TT in the case when no other cause could be found [9].

Thyroid-associated TT exacerbates the clinical course of comorbidities and long-term outcomes [47, 60] such as autoimmune hypothyroidism, which occurs in patients of all ages and with any severity of COVID-19 [5, 18, 26, 31, 57]. Uncontrolled TT could lead to more serious complications from SARS-CoV-2 infection [57, 61]. However, a meta-analytical study examining the relationship between the thyroid gland and the effects of COVID-19 in 31,339 patients with adverse events such as higher disease severity, ICU admission, mortality, and hospitalization showed that hypothyroidism was among the thyroid abnormalities, not TT, increased the risk of adverse effects of COVID-19 and depended on the patient's age [62].

Licensed in several countries vaccines against COVID-19 with different mechanisms of immunity have shown a satisfactory high level of safety and protection against this disease [24, 63]. Since the beginning of vaccination, there have been reports of thyroid dysfunction in the form of SAT and Graves' disease, which are the main etiological factors of TT, which determines the course of post-vaccination complications. GD is one of the diseases in autoimmune thyroiditis caused by autoantibodies that bind to antibodies to the $\mathrm{TSH}$ receptor (TSHR-Ab), which stimulates the thyroid gland to excess hormone production [64].

Regarding the formation of post-vaccination thyroid dysfunction, two possible mechanisms have been proposed. This is an autoimmune / inflammatory syndrome induced by vaccine adjuvants (ASIA), which are used as agents to increase immunogenicity and induce an adaptive immune response and cause adverse immune responses. Another mechanism is the autoimmune response after vaccination with COVID-19 through the adhesive glycoprotein SARS-CoV-2 [57, 65].

Most of the reported cases occurred after mRNA vaccination, although they were also caused by an inactivated vaccine. 
There are several cases of post-vaccination reactions that had certain features. Hraves' disease occurred after receiving the first and second doses of BNT162b2 mRNA vaccine in a 40 -year-old patient who had been observed for HT for 8 years [64]. Schimmel, J. et al. (2021) presented the first description of thyroiditis associated with SARS-CoV-2 vaccination of a 57-year-old woman who received the first dose of Pfizer-BioNTech SARS-CoV-2 vaccine 34 days before complaints and SAT clinics [66].

In three patients, signs of SAT with TT appeared 10 to 20 days after vaccination with Pfizer Bio-Ntech or Covid-19 Moderna mRNA vaccine. One of them had significantly increased erythrocyte sedimentation rate and IL-6 levels [66]. Bornemann, C. et al. (2021) reported two cases of cytologically confirmed SAT 2 weeks after receiving the first dose of the vaccine with Spikevax (Moderna Biotech, Spain) and Vaxzevria (AstraZeneca) [67]. One of the patients described [28] had no symptoms prior to vaccination, a nasopharyngeal smear test for SARS-CoV-2 and other SAT-related respiratory viruses was negative, and fever and thyroid pain appeared due to two weeks after vaccination with BNT162B2 mRNA (Pfizer-BioNTech). In another, biochemical and imaging features consistent with SAT were detected three weeks after vaccination with ChAdOx1-S (AstraZeneca). Two months after the onset of the reaction, both patients were euthyroid and asymptomatic [28]. Two mRNA vaccinated women with SARS-CoV-2 vaccine developed clinical manifestations of thyroid hyperactivity with elevated hormone levels after 3 days, which met the diagnostic criteria for Asian syndrome due to adjuvant (vaccine) exposure. Within a few days, they developed clinical manifestations of TT with the advent of antithyroid antibodies, i.e., the development of GD was associated with SARS-CoV-2 vaccination [68].

Described 2 cases of post-vaccination disorders, the first signs of which occurred in women 32 years $(3$ days after the second dose of Comirnaty (Pfizer BioNtech) and 33 years of age the day after the first dose of Pfizer. The onset of the reaction was dominated by neck pain and asthenia, weakness and hyperthermia. Based on the data of laboratory and ultrasound examination, which revealed a thyroid gland of heterogeneous structure and moderately increased vascularization, diagnosed with SAT. Normalization of the thyroid profile in the first patient occurred after 4 weeks. Asymptomatic condition and significant improvement in blood test in the second patient were found after two months. The thyroid gland remained slightly enlarged, mostly hypoechoic, with intensely increased vascularization. The patient was examined a month later and found healthy [69].

Of interest is the development of SAT after the use of inactivated vaccines, including reports of postvaccination reactions to the inactivated vaccine against SarsCoV-2 CoronaVac (Sinovac Life Sciences, China) in 3 patients who complained of pain in the front of the neck and fatigue after 4-7 days after vaccination. Clinical, laboratory and imaging features of their condition corresponded to SAT. These cases of SAT are regarded as a postvaccination phenomenon with ASIA syndrome, which occurs after exposure to adjuvants in vaccines and could cause inflammation and enhance immune responses $[65,70,71]$.

A 67-year-old man was hospitalized for fever, weight loss, and neck pain 18 days after the second dose of CoronaVac ${ }^{\circledR}$ inactivated vaccine. Laboratory results: TSH $<0.005 \mu \mathrm{IU} / \mathrm{ml}$ ), T3 $8.06 \mathrm{pg} / \mathrm{ml}$, T4 $2.87 \mathrm{ng} / \mathrm{DL})$. Ultrasound examination of the thyroid gland: increased size of the gland and its heterogeneous echotexture compatible with SAT [72, 73].

González LópezJ. Et al. (2021) draws attention to the complaints to be considered in patients with anterior cervical pain and general symptoms with recent SARSCoV-2 vaccination, emphasizing that this diagnosis is confirmed with three-phase evolution and ultrasound data $[10,70]$.

\section{Conclusions}

1. The prevalence of TD in patients with COVID19 varies from 13 to $64 \%$.

2 . The presence of thyroid dysfunction is positively correlated with the clinical severity of COVID-19

3. Patients with confirmed thyroid disease should receive COVID-19 vaccine to reduce the risk of morbidity and mortality from COVID-19 infection.

\section{Conflict of interests}

The authors declare that they have no conflicts of interest.

\section{Funding}

The study was performed without financial support.

\section{References}

1. Chakraborty, U., Ghosh, S., Chandra, A., Ray, A. K. (2020). Subacute thyroiditis as a presenting manifestation of COVID19: a report of an exceedingly rare clinical entity. BMJ Case Reports, 13 (12), e239953. doi: http://doi.org/10.1136/bcr-2020-239953

2. Schimmel, J., Alba, E. L., Chen, A., Russell, M., Srinath, R. (2021). Letter to the Editor: Thyroiditis and Thyrotoxicosis After the SARS-CoV-2 mRNA Vaccine. Thyroid, 31 (9), 1440-1440. doi: http://doi.org/10.1089/thy.2021.0184

3. Wu, Z., McGoogan, J. M. (2020). Characteristics of and Important Lessons From the Coronavirus Disease 2019 (COVID19) Outbreak in China. JAMA, 323 (13), 1239-1242. doi: http://doi.org/10.1001/jama.2020.2648

4. Kim, S. Y., Kim, D. W. (2020). Does the Clinical Spectrum of Coronavirus Disease 2019 (COVID-19) Show Regional Differences? Clinical and Experimental Otorhinolaryngology, 13 (2), 83-84. doi: http://doi.org/10.21053/ceo.2020.00612

5. Trimboli, P., Cappelli, C., Croce, L., Scappaticcio, L., Chiovato, L., Rotondi, M. (2021). COVID-19-Associated Subacute Thyroiditis: Evidence-Based Data From a Systematic Review. Frontiers in Endocrinology, 12. doi: http://doi.org/10.3389/fendo.2021.707726

6. Huang, C., Wang, Y., Li, X., Ren, L., Zhao, J., Hu, Y. et. al. (2020). Clinical features of patients infected with 2019 novel coronavirus in Wuhan, China. The Lancet, 395 (10223), 497-506. doi: http://doi.org/10.1016/s0140-6736(20)30183-5

7. A Abobaker, A., Darrat, M. (2021). The association between biochemically confirmed thyroid gland disorder and morbidity and mortality in patients with COVID-19. Journal of Medical Virology, 93 (12), 6449-6450. doi: http://doi.org/10.1002/jmv.27213 
8. Zhou, F., Yu, T., Du, R., Fan, G., Liu, Y., Liu, Z. et. al. (2020). Clinical course and risk factors for mortality of adult inpatients with COVID-19 in Wuhan, China: a retrospective cohort study. The Lancet, 395 (10229), 1054-1062. doi: http://doi.org/10.1016/s01406736(20)30566-3

9. Edwards, K., Hussain, I. (2021). Two Cases of Severe Autoimmune Thyrotoxicosis Following SARS-CoV-2 Infection. Journal of Investigative Medicine High Impact Case Reports, 9. doi: http://doi.org/10.1177/23247096211056497

10. Kim, S.-Y., Yoo, D.-M., Min, C.-Y., Choi, H.-G. (2021). The Effects of Previous Thyroid Disease on the Susceptibility to, Morbidity of, and Mortality Due to COVID-19: A Nationwide Cohort Study in South Korea. Journal of Clinical Medicine, 10 (16), 3522. doi: http://doi.org/10.3390/jcm10163522

11. Bakshi, S. S., Kalidoss, V. K. (2021). Is there an association between hypothyroidism and COVID 19?: A preliminary report. Wiener klinische Wochenschrift, 133 (7-8),414-415. doi: http://doi.org/10.1007/s00508-021-01813-2

12. Dworakowska, D., Grossman, A. B. (2020). Thyroid disease in the time of COVID-19. Endocrine, 68 (3),471-474. doi: http://doi.org/10.1007/s12020-020-02364-8

13. Sanyaolu, A., Okorie, C., Marinkovic, A., Patidar, R., Younis, K., Desai, P. et. al. (2020). Comorbidity and its Impact on Patients with COVID-19. SN Comprehensive Clinical Medicine, 2 (8), 1069-1076. doi: http://doi.org/10.1007/s42399-020-00363-4

14. Dosi, R., Jain, G., Mehta, A. (2020). Clinical Characteristics, Comorbidities, and Outcome among 365 Patients of Coronavirus Disease2019at a Tertiary Care Centre in Central India. The Journal of the Association of Physicians of India, 68 (9),20-23.

15. Van Gerwen, M., Alsen, M., Little, C., Barlow, J., Naymagon, L., Tremblay, D. et. al. (2020). Outcomes of Patients With Hypothyroidism and COVID-19: A Retrospective Cohort Study. Frontiers in Endocrinology, 11. doi: http://doi.org/10.3389/fendo.2020.00565

16. Brojakowska, A., Eskandari, A., Bisserier, M., Bander, J., Garikipati, V. N. S., Hadri, L. et. al. (2021). Comorbidities, sequelae, blood biomarkers and their associated clinical outcomes in the Mount Sinai Health System COVID-19 patients. PLOS ONE, 16 (7), e0253660. doi: http://doi.org/10.1371/journal.pone.0253660

17. Brix, T. H., Hegedüs, L., Hallas, J., Lund, L. C. (2021). Risk and course of SARS-CoV-2 infection in patients treated for hypothyroidism and hyperthyroidism. The Lancet Diabetes \& Endocrinology, 9 (4), 197-199. doi: http://doi.org/10.1016/s22138587(21)00028-0

18. Duntas, L. H., Jonklaas, J. (2021). COVID-19 and Thyroid Diseases: A Bidirectional Impact. Journal of the Endocrine Society, 5 (8). doi: http://doi.org/10.1210/jendso/bvab076

19. Hariyanto, T. I., Kurniawan, A. (2020). Thyroid disease is associated with severe coronavirus disease 2019 (COVID-19) infection. Diabetes \& Metabolic Syndrome: Clinical Research \& Reviews, 14 (5), 1429-1430. doi: http://doi.org/10.1016/j.dsx.2020.07.044

20. Lisco, G., De Tullio, A., Jirillo, E., Giagulli, V. A., De Pergola, G., Guastamacchia, E., Triggiani, V. (2021). Thyroid and COVID-19: a review on pathophysiological, clinical and organizational aspects. Journal of Endocrinological Investigation, 44 (9), 1801-1814. doi: http://doi.org/10.1007/s40618-021-01554-z

21. Giovanella, L., Ruggeri, R. M., Ovčariček, P. P., Campenni, A., Treglia, G., Deandreis, D. (2021). Prevalence of thyroid dysfunction in patients with COVID-19: a systematic review. Clinical and Translational Imaging, 9 (3), 233-240. doi: http://doi.org/10.1007/ s40336-021-00419-y

22. Khoo, B., Tan, T., Clarke, S. A., Mills, E. G., Patel, B., Modi, M. et. al. (2020). Thyroid Function Before, During, and After COVID-19. The Journal of Clinical Endocrinology \& Metabolism, 106 (2), e803-e811. doi: http://doi.org/10.1210/clinem/dgaa830

23. Ippolito, S., Dentali, F., Tanda, M. L. (2020). SARS-CoV-2: a potential trigger for subacute thyroiditis? Insights from a case report. Journal of Endocrinological Investigation, 43 (8), 1171-1172. doi: http://doi.org/10.1007/s40618-020-01312-7

24. Lui, D. T. W., Lee, C. H., Chow, W. S., Lee, A. C. H., Tam, A. R., Fong, C. H. Y. et. al. (2020). Thyroid Dysfunction in Relation to Immune Profile, Disease Status, and Outcome in 191 Patients with COVID-19. The Journal of Clinical Endocrinology \& Metabolism, 106 (2), e926-e935. doi: http://doi.org/10.1210/clinem/dgaa813

25. Sen, K., Sinha, A., Sen, S., Chakraborty, S., Alam, M. (2020). Thyroid Function Test in COVID-19 Patients: A Cross-Sectional Study in a Tertiary Care Hospital. Indian Journal of Endocrinology and Metabolism, 24 (6), 532-536. doi: http://doi.org/10.4103/ ijem.ijem_779_20

26. Caron, P. (2020). Thyroid disorders and SARS-CoV-2 infection: From pathophysiological mechanism to patient management. Annales d'Endocrinologie, 81 (5), 507-510. doi: http://doi.org/10.1016/j.ando.2020.09.001

27. Sandru, F., Carsote, M., Petca, R., Gheorghisan-Galateanu, A., Petca, A., Valea, A., Dumitrascu, M. (2021). COVID-19 related thyroid conditions (Review). Experimental and Therapeutic Medicine, 22 (1). doi: http://doi.org/10.3892/etm.2021.10188

28. Siolos, A., Gartzonika, K., Tigas, S. (2021). Thyroiditis following vaccination against COVID-19: Report of two cases and review of the literature. Metabolism Open, 12, 100136. doi: http://doi.org/10.1016/j.metop.2021.100136

29. Trimboli, P., Camponovo, C., Scappaticcio, L., Bellastella, G., Piccardo, A., Rotondi, M. (2021). Thyroid sequelae of COVID19: a systematic review of reviews. Reviews in Endocrine and Metabolic Disorders, 22 (2), 485-491. doi: http://doi.org/10.1007/s11154021-09653-1

30. Daraei, M., Hasibi, M., Abdollahi, H., Mirabdolhagh Hazaveh, M., Zebaradst, J., Hajinoori, M., Asadollahi-Amin, A. (2020). Possible role of hypothyroidism in the prognosis of COVID- 19. Internal Medicine Journal, 50 (11), 1410-1412. doi: http://doi.org/10.1111/imj.15000

31. Speer, G., Somogyi, P. (2021). Thyroid complications of SARS and coronavirus disease 2019 (COVID-19). Endocrine Journal, 68 (2), 129-136. doi: http://doi.org/10.1507/endocrj.ej20-0443

32. Martins, J. R. M., Villagelin, D. G. P., Carvalho, G. A., Vaisman, F., Teixeira, P. F. S., Scheffel, R. S., Sgarbi, J. A. (2021). Management of thyroid disorders during the COVID-19 outbreak: a position statement from the Thyroid Department of the Brazilian Society of Endocrinology and Metabolism (SBEM). Archives of Endocrinology and Metabolism, 65 (3), 368-375. doi: http://doi.org/10.20945/2359-3997000000352

33. Pereira, D. N., Gontijo Silveira, L. F., Moreira Guimarães, M. M., Polanczyk, C. A., Sousa Nunes, A. G., de Moura Costa, A. S. et. al. (2021). Hypothyroidism does not lead to worse prognosis in COVID-19: findings from the Brazilian COVID-19 registry. doi: http://doi.org/10.1101/2021.11.03.21265685

34. Novel Coronavirus (COVID-19) and the Thyroid: Frequently Asked Questions. Available at: https://www.thyroid.org/covid-19/ coronavirus-frequently-questions/

35. Montesinos, M. del M., Pellizas, C. G. (2019). Thyroid Hormone Action on Innate Immunity. Frontiers in Endocrinology, 10. doi: http://doi.org/10.3389/fendo.2019.00350 
36. McKechnie, J. L., Blish, C. A. (2020). The Innate Immune System: Fighting on the Front Lines or Fanning the Flames of COVID-19? Cell Host \& Microbe, 27 (6), 863-869. doi: http://doi.org/10.1016/j.chom.2020.05.009

37. Velavan, T. P., Meyer, C. G. (2020). Mild versus severe COVID-19: Laboratory markers. International Journal of Infectious Diseases, 95, 304-307. doi: http://doi.org/10.1016/j.ijid.2020.04.061

38. Abobaker, A., Raba, A. A., Alzwi, A. (2020). Extrapulmonary and atypical clinical presentations of COVID- 19. Journal of Medical Virology, 92 (11), 2458-2464. doi: http://doi.org/10.1002/jmv.26157

39. Chen, M., Zhou, W., Xu, W. (2021). Thyroid Function Analysis in 50 Patients with COVID-19: A Retrospective Study. Thyroid, 31 (1), 8-11. doi: http://doi.org/10.1089/thy.2020.0363

40. Gorini, F., Bianchi, F., Iervasi, G. (2020). COVID-19 and Thyroid: Progress and Prospects. International Journal of Environmental Research and Public Health, 17 (18), 6630. doi: http://doi.org/10.3390/ijerph17186630

41. Lang, S., Liu, Y., Qu, X., Lu, R., Fu, W., Zhang, W. et. al. (2021). Association between Thyroid Function and Prognosis of COVID-19: A Retrospective Observational Study. Endocrine Research, 46 (4), 170-177. doi: http://doi.org/10.1080/07435800. 2021.1924770

42. Muller, I., Cannavaro, D., Dazzi, D., Covelli, D., Mantovani, G., Muscatello, A. et. al. (2020). SARS-CoV-2-related atypical thyroiditis. The Lancet Diabetes \& Endocrinology, 8 (9), 739-741. doi: http://doi.org/10.1016/s2213-8587(20)30266-7

43. Baldelli, R., Nicastri, E., Petrosillo, N., Marchioni, L., Gubbiotti, A., Sperduti, I. et. al. (2021). Thyroid dysfunction in COVID-19 patients. Journal of Endocrinological Investigation, 44 (12), 2735-2739. doi: http://doi.org/10.1007/s40618-021-01599-0

44. Stasiak, M., Lewiński, A. (2021). New aspects in the pathogenesis and management of subacute thyroiditis. Reviews in Endocrine and Metabolic Disorders, 22 (4), 1027-1039. doi: http://doi.org/10.1007/s11154-021-09648-y

45. Mateu-Salat, M., Urgell, E., Chico, A. (2020). SARS-COV-2 as a trigger for autoimmune disease: report of two cases of Graves' disease after COVID-19. Journal of Endocrinological Investigation, 43 (10), 1527-1528. doi: http://doi.org/10.1007/s40618020-01366-7

46. Khatri, A., Charlap, E., Kim, A. (2020). Subacute Thyroiditis from COVID-19 Infection: A Case Report and Review of Literature. European Thyroid Journal, 9 (6), 324-328. doi: http://doi.org/10.1159/000511872

47. Ruggeri, R. M., Campennì, A., Siracusa, M., Frazzetto, G., Gullo, D. (2020). Subacute thyroiditis in a patient infected with SARS-COV-2: an endocrine complication linked to the COVID-19 pandemic. Hormones, 20 (1), 219-221. doi: http://doi.org/10.1007/ s42000-020-00230-w

48. Whiting, A., Reyes, J. V. M., Ahmad, S., Lieber, J. (2021). Post-COVID-19 Fatigue: A Case of Infectious Hypothyroidism. Cureus, 13 (5). doi: http://doi.org/10.7759/cureus.14815

49. Inaba, H., Aizawa, T. (2021). Coronavirus Disease 2019 and the Thyroid - Progress and Perspectives. Frontiers in Endocrinology, 12. doi: http://doi.org/10.3389/fendo.2021.708333

50. Brancatella, A., Ricci, D., Cappellani, D., Viola, N., Sgrò, D., Santini, F., Latrofa, F. (2020). Is Subacute Thyroiditis an Underestimated Manifestation of SARS-CoV-2 Infection? Insights From a Case Series. The Journal of Clinical Endocrinology \& Metabolism, 105 (10), e3742-e3746. doi: http://doi.org/10.1210/clinem/dgaa537

51. Brancatella, A., Ricci, D., Viola, N., Sgrò, D., Santini, F., Latrofa, F. (2020). Subacute Thyroiditis After Sars-COV-2 Infection. The Journal of Clinical Endocrinology \& Metabolism, 105 (7), 2367-2370. doi: http://doi.org/10.1210/clinem/dgaa276

52. Malik, J., Zaidi, S. M. J., Waqar, A. U., Khawaja, H., Malik, A., Ishaq, U. et. al. (2021). Association of hypothyroidism with acute COVID-19: a systematic review. Expert Review of Endocrinology \& Metabolism, 16 (5), 251-257. doi: http://doi.org/10.1080/ 17446651.2021 .1968830

53. Liu, J., Wu, X., Lu, F., Zhao, L., Shi, L., Xu, F. (2016). Low T3 syndrome is a strong predictor of poor outcomes in patients with community-acquired pneumonia. Scientific Reports, 6 (1). doi: http://doi.org/10.1038/srep22271

54. Güven, M., Gültekin, H. (2021). The prognostic impact of thyroid disorders on the clinical severity of COVID- 19: Results of single-centre pandemic hospital. International Journal of Clinical Practice, 75 (6). doi: http://doi.org/10.1111/ijcp.14129

55. Li, X., Marmar, T., Xu, Q., Tu, J., Yin, Y., Tao, Q. et. al. (2020). Predictive indicators of severe COVID-19 independent of comorbidities and advanced age: a nested case-control study. Epidemiology and Infection, 148. doi: http://doi.org/10.1017/ s0950268820002502

56. Fliers, E., Bianco, A. C., Langouche, L., Boelen, A. (2015). Thyroid function in critically ill patients. The Lancet Diabetes \& Endocrinology, 3 (10), 816-825. doi: http://doi.org/10.1016/s2213-8587(15)00225-9

57. Boelaert, K., Visser, W. E., Taylor, P. N., Moran, C., Léger, J., Persani, L. (2020). Endocrinology in the time of COVID-19: Management of hyperthyroidism and hypothyroidism. European Journal of Endocrinology, 183 (1), G33-G39. doi: http://doi.org/10.1530/ eje-20-0445

58. Garg, S., Dabas, A., Singh, H., Goswami, B., Kumar, K., Dubey, A. et. al. (2021). Thyroid dysfunction in COVID-19. Indian Journal of Endocrinology and Metabolism, 25 (3), 198-201. doi: http://doi.org/10.4103/ijem.ijem_195_21

59. Lania, A., Sandri, M. T., Cellini, M., Mirani, M., Lavezzi, E., Mazziotti, G. (2020). Thyrotoxicosis in patients with COVID-19: the THYRCOV study. European Journal of Endocrinology, 183 (4), 381-387. doi: http://doi.org/10.1530/eje-20-0335

60. Chen, W., Tian, Y., Li, Z., Zhu, J., Wei, T., Lei, J. (2021). Potential Interaction Between SARS-CoV-2 and Thyroid: A Review. Endocrinology, 162 (3). doi: http://doi.org/10.1210/endocr/bqab004

61. BTA/SFE statement regarding issues specific to thyroid dysfunction during the COVID-19 pandemic (2021). Available at: https://www.british-thyroid-association.org/sandbox/bta2016/management-of-thyroid-dysfunction-during-covid-19_final.pdf

62. Damara, F. A., Muchamad, G. R., Ikhsani, R., Hendro, Syafiyah, A. H., Bashari, M. H. (2021). Thyroid disease and hypothyroidism are associated with poor COVID-19 outcomes: A systematic review, meta-analysis, and meta-regression. Diabetes \& Metabolic Syndrome: Clinical Research \& Reviews, 15 (6), 102312. doi: http://doi.org/10.1016/j.dsx.2021.102312

63. Lui, D., Lee, K. K., Lee, C. H., Lee, A., Hung, I., Tan, K. (2021). Development of Graves' Disease After SARS-CoV-2 mRNA Vaccination: A Case Report and Literature Review. Frontiers in public health, 9, 778964. doi: http://doi.org/10.3389/fpubh.2021.778964

64. Bornemann, C., Woyk, K., Bouter, C. (2021). Case Report: Two Cases of Subacute Thyroiditis Following SARS-CoV-2 Vaccination. Frontiers in Medicine, 8. doi: http://doi.org/10.3389/fmed.2021.737142

65. Schimmel, J., Alba, E. L., Chen, A., Russell, M., Srinath, R. (2021). Letter to the Editor: Thyroiditis and Thyrotoxicosis After the SARS-CoV-2 mRNA Vaccine. Thyroid, 31 (9), 1440-1440. doi: http://doi.org/10.1089/thy.2021.0184

66. Watad, A., Sharif, K., Shoenfeld, Y. (2017). The ASIA syndrome: basic concepts. Mediterranean Journal of Rheumatology, 28 (2), 64-69. doi: http://doi.org/10.31138/mjr.28.2.64 
67. Stasiak, M., Lewiński, A. (2021). New aspects in the pathogenesis and management of subacute thyroiditis. Reviews in Endocrine and Metabolic Disorders, 22 (4), 1027-1039. doi: http://doi.org/10.1007/s11154-021-09648-y

68. Velavan, T. P., Meyer, C. G. (2020). Mild versus severe COVID-19: Laboratory markers. International Journal of Infectious Diseases, 95, 304-307. doi: http://doi.org/10.1016/j.ijid.2020.04.061

69. Vera-Lastra, O., Ordinola Navarro, A., Cruz Domiguez, M. P., Medina, G., Sánchez Valadez, T. I., Jara, L. J. (2021). Two Cases of Graves' Disease Following SARS-CoV-2 Vaccination: An Autoimmune/Inflammatory Syndrome Induced by Adjuvants. Thyroid, 31 (9), 1436-1439. doi: http://doi.org/10.1089/thy.2021.0142

70. González López, J., Martín Niño, I., Arana Molina, C. (2021). Tiroiditis subaguda tras vacunación contra el SARS-CoV2: a propósito de dos casos. Medicina Clínica. doi: http://doi.org/10.1016/j.medcli.2021.11.002

71. İremli, B. G., Şendur, S. N., Ünlütürk, U. (2021). Three Cases of Subacute Thyroiditis Following SARS-CoV-2 Vaccine: Postvaccination ASIA Syndrome. The Journal of Clinical Endocrinology \& Metabolism, 106 (9), 2600-2605. doi: http://doi.org/10.1210/ clinem/dgab373

72. Şahin Tekin, M., Şaylısoy, S., Yorulmaz, G. (2021). Subacute thyroiditis following COVID-19 vaccination in a 67-year-old male patient: a case report. Human Vaccines \& Immunotherapeutics, 17 (11), 4090-4092. doi: http://doi.org/10.1080/21645515.2021.1947102

73. Dutta, A., Jevalikar, G., Sharma, R., Farooqui, K. J., Mahendru, S., Dewan, A. et. al. (2021). Low FT3 is an independent marker of disease severity in patients hospitalized for COVID-19. Endocrine Connections, 10 (11), 1455-1462. doi: http://doi.org/10.1530/ ec-21-0362

Received date 17.11.2021

Accepted date 24.12.2021

Published date 30.01.2022

Daria Korchagina*, PhD, State Institution "V. Danilevsky Institute for Endocrine Pathology Problems of the National Academy of Medical Sciences of Ukraine", Alchevskykh str., 10, Kharkiv, Ukraine, 61002

Iegor Korchagin, PhD, Medical Diagnostic Centre «RISHON CLINIC», Pischana str., 11, Kharkiv, Ukraine, 62419

*Corresponding author: Daria Korchagina, e-mail: korchagina.daria@ukr.net 